\author{
M. E. Diken ${ }^{1}$, S. Doğan*², Y. Turhan ${ }^{3}$ and M. Doğan ${ }^{3}$ \\ ${ }^{1}$ Balikesir University, Science and Technology Application and Research Center, Turkey \\ ${ }^{2}$ Balikesir University Faculty of Science and Literature Department of Molecular Biology and \\ Genetics, Turkey \\ ${ }^{3}$ Balikesir University Faculty of Science and Literature Department of Chemistry, Turkey \\ *sdogan@balikesir.edu.tr
}

\title{
SYNTHESIS AND CHARACTERIZATION OF POLY(ACRYLIC ACID)/ORGANO-MODIFIED NANOHYDROXYAPATITE NANOCOMPOSITES: THERMAL, OPTICAL AND BIOCOMPATIBILITY PROPERTIES
}

\begin{abstract}
The aim of this study was to investigate the structural, thermal, optical and biocompatibility properties of poly(acrylic acid)(PAA)/organo-modified nanohydroxyapatite (OM-nHAp) nanocomposites synthesized by solvent intercalation method. The characterization of PAA/OM-nHAp nanocomposites was made by different techniques. SEM and TEM results showed that OM-nHAp particles were dispersed in the nanoscale into PAA matrix and that they were uniformly distributed within film. Glass transition temperature of PAA increased with OM-nHAp content. Ultraviolet (UV) absorbance experiments showed that PAA had a higher UV transmission than its nanocomposites. The biocompatibility of nanocomposites was also examined in simulated body fluid.
\end{abstract}

Keywords: Organo-modified nanohydroxyapatite, poly(acrylic acid), nanocomposite, characterization, biocompatibility.

\section{INTRODUCTION}

In recent years, the design and development of nanoparticles and nanostructure materials have opened a new era for constructing well designed nanostructures that have been considered as a novel class of materials for biomedical applications [1]. The various manmade biomaterials have offered a promising alternative approach to the bone treatment. These biomaterials must be biocompatible and meet certain minimal morphological and physicochemical properties to achieve a high degree of biocompatibility and biofunctionality [2]. The conventional materials for bone implant are titanium and its alloys. This is due to their favorable mechanical hardness and chemical stability. However, owning to their limited bioactivities, they cannot bind to the bone well and they fail to promote new bone formation on their surfaces during the early stages after implantation. The current design and fabrication of biomaterials used in bone regeneration involves a range of various materials based on ceramics and synthetic or natural polymers and their composites [3]. 
Polymer/inorganic nanocomposites hold substantial promise for the production of novel biomaterials. In the biomedical field, the synthesis of polymer/HAp composite materials is of great interest for the development of biomaterials suitable to repair the skeletal system. HAp has two important characteristics as biomaterials: biocompatibility and bioactivity [4]. It is widely used as bone replacement due to its excellent biocompatibility, bioactivity, and osteoconductive properties. These properties are given by its unique chemical composition and crystal structure that is similar to the human skeletal apatite [5]. However, as these materials possess some low physical properties such as thermal stability, application of synthetic hydroxyapatite has been restricted exclusively to some areas. A suitable method of improving its some properties is based on the synthesis of composites made of HAp and other ceramic powders, metal particles or polymers [6].

More important, the efficiency of a biomaterial, depends greatly on its ability to interact with cells, but little is known about this interaction for this kind of nanocomposite [3]. One of the main advantages of polymer/HAp composites with respect to HAp biomaterials is the possibility to modulate biodegradability, bioactivity, and thermal properties through variations in composition. Furthermore, the presence of the polymer could improve the interfacial bonding of the composite with bone tissue [7]. Poly(acrylic acid) (PAA), a water soluble thermoplastic polymer, has been used in numerous clinical applications. HAp is a major inorganic component that nearly formed $60 \%$ of human skeleton and has been used extensively for biomedical implant applications and bone regeneration due to its bioactive, biodegradable and osteoconductive properties [1].

There are numerous studies in the literature to improve the thermal, mechanical, optical and biocompatibility properties of polymers using HAp. However, improvements in these studies are not at the desired level, yet. Here are some of these works. Wosek (2005) synthsized the polyurethane/hydroxyapatite composites by using the solvent-casting salt leaching method [8]; Yilmaz et al. (2018) the PMMA/nHAp nanocomposites using different molecular weights of PMMA (120,000, 350,000, and 996,000 g/mol) and nHAp (1, 2.5 and $5 \%$ ) according to melt blending method, and determined the properties of hemocompatibility, cytotoxicity, and genotoxicity of PMMA/nHAp nanocomposites [9]; and Diken et al. (2018) poly(methylmethacrylate)/nanohydroxyapatite (PMMA/nHAp) nanocomposites, characterized by different methods, and then, determined their hemocompatibility and antbactrerial properties [10]. Yoshida et al. (2004) prepared chitosan-HAp nanocomposites through mechanochemical reaction using conventional ball mill and subsequent aging, and expected to be one of the useful bone substitute materials of the prepared chitosan-HAp composites [11]. Khan et al. (2013) synthesized polyurethane nanocomposite material by chemically binding nHAp to the diisocyanate component of the polyurethane backbone by solvent polymerization. They found that i. linkage between the hard and soft segments were urethane linkage, ii. nanocomposites showed high mechanical properties with increase in content of nHAp, and iii. polyurethane composites with nHAp exhibited biocompatibility with respect to mammalian cell growth [12]. Silva et al. (2001) prepared two series of zirconia-HAp composites by powder uniaxial pressing at $700 \mathrm{MPa}$ and sintering in air at 1200-1500 ${ }^{\circ} \mathrm{C}$ for $3 \mathrm{~h}$ [6]. Pon-on et al. (2013) investigated the physical and biocompatible properties of HAp composites having different amounts of titania rod in them (10-90 wt\%). They found that $i$. the synthesized powder showed a core-shell structure and ii. in vitro cell adhesion tests confirmed the osteoblast attachment and growth on the HApTiR's surface [3]. PAA/nHAp nanocomposites were also synthesized to improve the thermal and biocompatibility properties of polymers such as poly(acrylic acid), but were not at the desired level, yet. For example, El-Bahy et al. (2011) synthesized biocomposite films of HAp and poly(acrylic acid) with different amounts of HAp nanoparticles. The results showed that the 
biocomposites were formed with good homogeneity and thermal stability. Nanoparticles of HAp were uniformly distributed in the polymeric matrices. The resulting new materials were hoped to be applicable in the biomedical fields [13].

As seen from the above studies, there are limited study to improve the interaction and biocompatibility between poly(acrylic acid) and HAp. Therefore, the purpose of the present investigation was to synthesize and characterize the biocompatible poly(acrylic acid) (PAA)/organo-modified nHAp nanocomposites using solvent intercalating method. Solvent intercalation is a nanocomposite preparing method, which is provided the dispersion of the filler material using solvent and sonication processes. And then, interacting with the polymer is happen. This method is preferred in the literature especially when preparing polymer/clay and polymer/CNT nanocomposites. In this method, the essential point is the solvent choice. The chosen solvent should well disperse the filler material and well dissolve the polymer, homogenously $[14,15]$. In literature, there are not any works related to PAA/organo-modified nHAp nanocomposites after organo modification of hydroxyapatite surfaces. This is the first work done in this area. The structures of the prepared nanocomposites were characterized using FTIR-ATR, XRD, SEM, TEM, TG, DSC and UV-Visible spectrophotometer. Their bioactivities were also examined in simulated body fluid (SBF) that had similar concentrations to human blood plasma.

\section{MATERIALS AND METHODS}

\section{Materials}

PAA, average molecular weight 450 000, [3-(2-aminoethylamino)propyl]trimethoxysilane (3-APT), $\quad \mathrm{N}^{1}$-[3-(trimethoxysilylpropyl)]-diethiltriamin (N-3TMPEDA), 3-(chloropropyl)trimethoxysilane (3-CPTMS), toluen, methanol and acetone were purchased from Sigma-Aldrich. All the chemicals were analytical grade and used without further purification.

$1 \mathrm{~g}$ dry nHAp was suspended in $100 \mathrm{~mL}$ of toluene, then, $10 \mathrm{mmol}$ of silane compounds were added into this suspension, and the reaction mixture was refluxed for $6 \mathrm{~h}$. OM-nHAp was filtered, washed with toluene and diethyl ether, and then, dried in vacuum oven overnight. This process was made one by one to each organosilane compounds such as 3APT, N-3-TMPEDA and 3-CPTMS [16,17].

Certain amount of OM-nHAp particles contained in a $250 \mathrm{~mL}$ reaction vessel was added to $50 \mathrm{~mL}$ of solvent (distilled water) and the suspension was mixed for $20 \mathrm{~min}$ in an ultrasonic bath. At the same time, $1 \mathrm{~g}$ PAA and $50 \mathrm{~mL}$ solvent were added into another reaction vessel and mixture was mixed to completely dissolve PAA at magnetic stirrer for $2 \mathrm{~h}$. Then, PAA and OM-nHAp suspensions were combined and mixed on the magnetic stirrer at room temperature for $24 \mathrm{~h}$. The solvent was removed in vacuum oven. Eventually, PAA/OM-nHAp nanocomposite films were obtained [18-20].

\section{Methods}

FTIR-ATR analysis of samples was carried out by PerkinElmer Spectrum 100 FTIRATR in the wavelength ranges of 4000 to $650 \mathrm{~cm}^{-1}$ in the transmission mode.

X-ray diffraction analysis for samples was carried out on a Analytical Philips X'Pert-Pro $\mathrm{X}$-ray diffractometer equipped with a back monochromator operating at $40 \mathrm{kV}$ and a copper 
cathode as the $\mathrm{X}$-ray source $(\lambda=1.54 \AA)$ at room temperature. The analysis rate was $2{ }^{\circ} \mathrm{C} / \mathrm{min}$ over a range of $5-50^{\circ} \mathrm{C}$.

Thermogravimetric analysis (TG) were performed using PerkinElmer Diamond simultaneous DTA/TG instrument with a heating rate of $10^{\circ} \mathrm{C} / \mathrm{min}$ from $25^{\circ} \mathrm{C}$ to $600^{\circ} \mathrm{C}$ under nitrogen atmosphere.

Glass transition temperatures were determined at a temperature rate of $10^{\circ} \mathrm{C} / \mathrm{min}$ from room temperature to $200^{\circ} \mathrm{C}$ under $\mathrm{N}_{2}$ atmosphere by PerkinElmer DSC 4000 .

The UV-Vis spectra of PAA and its nanocomposites in the wavelength ranges of 200-700 nm were measured using a UV-Vis spectrophotometer (PerkinElmer Lamda 35).

SEM and TEM measurements of PAA/OM-nHAp nanocomposites were performed by a Neoscope JCM-5000 BENCHTOP-SEM and a high-resolution FEI Tecnai G2 F30 transmission electron microscope, respectively. Most of the measurements were carried out using an accelerating voltage of $200 \mathrm{kV}$. The OM-nHAp powders and corresponding nanocomposites were suspended in water and a drop of the resultant mixture was deposited on a carbon grid and dried in oven.

Hemocompatibility of PAA and its nanocomposites was determined by spectrophotometric process. Human blood $(2 \mathrm{~mL})$ was stored in citrated tubes against coagulation at $4^{\circ} \mathrm{C}$. Blood samples were diluted with salt solution. To positive control, the samples were diluted by adding of $0.2 \mathrm{~mL}$ of blood and $0.5 \mathrm{~mL}$ of $0.01 \mathrm{M} \mathrm{HCl}$, and then the sample completed to $10 \mathrm{~mL}$ was incubated at $37^{\circ} \mathrm{C}$ for $1 \mathrm{~h}$. To negative control, $0.2 \mathrm{~mL}$ of blood sample was completed to $10 \mathrm{~mL}$ with normal salt solution, and then, incubated at $37^{0} \mathrm{C}$ for $1 \mathrm{~h}$. The nanocomposites, which had sharp endless $5 \times 5 \mathrm{~mm}(0,0022 \mathrm{~g})$ dimensions, were transferred to the blood sample in centrifuge tube and incubated at $37^{\circ} \mathrm{C}$ for $1 \mathrm{~h}$. After incubation, all sample solution was centrifuged for $10 \mathrm{~min}$ at $6000 \mathrm{rpm}$, and the supernatant was analyzed spectrophotometrically. If hemolysis $\%$ is $5 \%$ and below $5 \%$, nanocomposites is hemocompatibility. If hemolysis $\%$ is $10 \%$ and below $10 \%$, it is a biocompatibility nanocomposite [21].

\section{RESULTS AND DISCUSSION}

\section{FTIR-ATR spectra of nHAp/modified-nHAp and their bionanocomposites}

FTIR-ATR spectrum in Figure 1 has shown the characteristic absorption peaks of nHAp and OM-nHAp nanoparticles. The bands at 3374 and $1641 \mathrm{~cm}^{-1}$ were attributed to adsorbed water, while the peak at $3569 \mathrm{~cm}^{-1}$ was attributable to the stretching vibration of the lattice $\mathrm{OH}^{-}$ions. The characteristic band for $\mathrm{PO}_{4}{ }^{3-}$ appeared at 1088,1015 and $961 \mathrm{~cm}^{-1}$. The observation of the asymmetric P-O stretching vibration of the $\mathrm{PO}_{4}{ }^{3-}$ band at $961 \mathrm{~cm}^{-1}$ as a distinguishable peak corresponded to the triply degenerate bending vibrations of $\mathrm{PO}_{4}{ }^{3-}$ in HAp. These results were similar to those reported in the literature [22]. FTIR-ATR spectra of PAA in Figure 2 has shown the characteristic stretching band of carboxylic group $\mathrm{C}=\mathrm{O}$ at $1693 \mathrm{~cm}^{-1}$, $\mathrm{CH}$ stretch band between $3000-2800 \mathrm{~cm}^{-1}, \mathrm{CH}_{2}$ bending band at $1451 \mathrm{~cm}^{-1}$, coupling between $\mathrm{OH}$ and $\mathrm{CO}$ of in-plane $\mathrm{OH}$ bending and $\mathrm{CO}$ stretching of neighboring, and $\mathrm{C}-\mathrm{O}$ band at about 1162 and $1046 \mathrm{~cm}^{-1}$ [23]. 

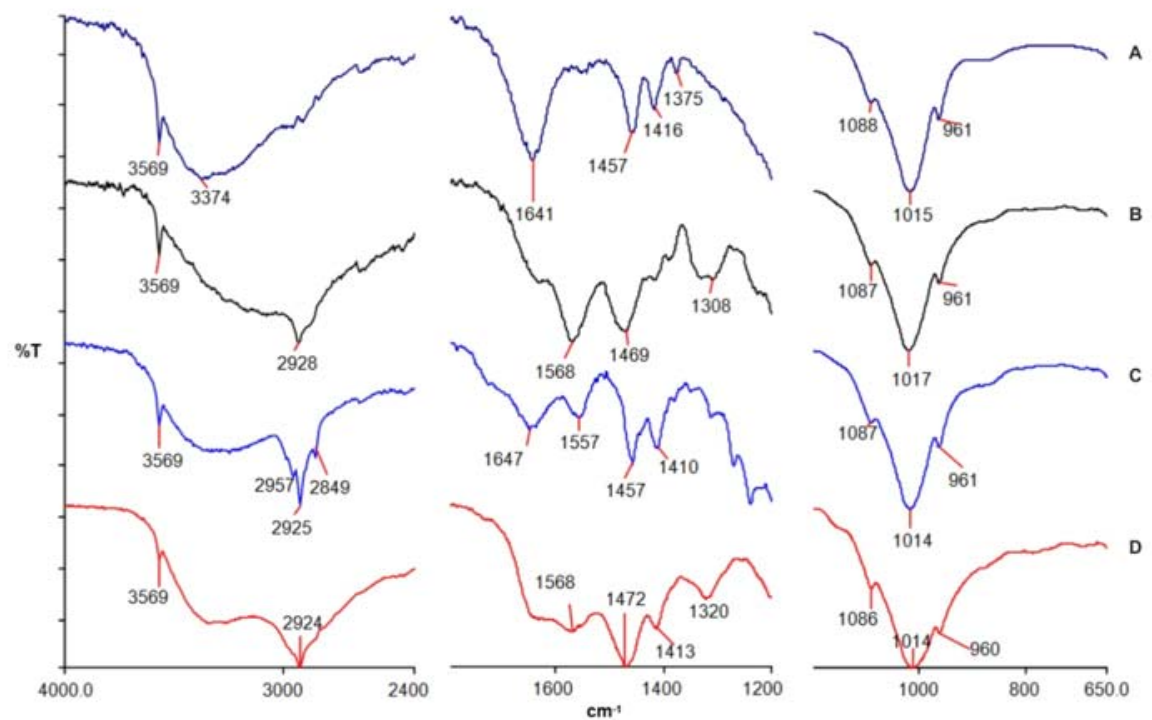

Fig.1. FTIR-ATR spectra of A. nHAp, B. 3-APT-nHAp, C. 3-CPTMS-nHAp, D. N-3-TMPEDA-nHAp

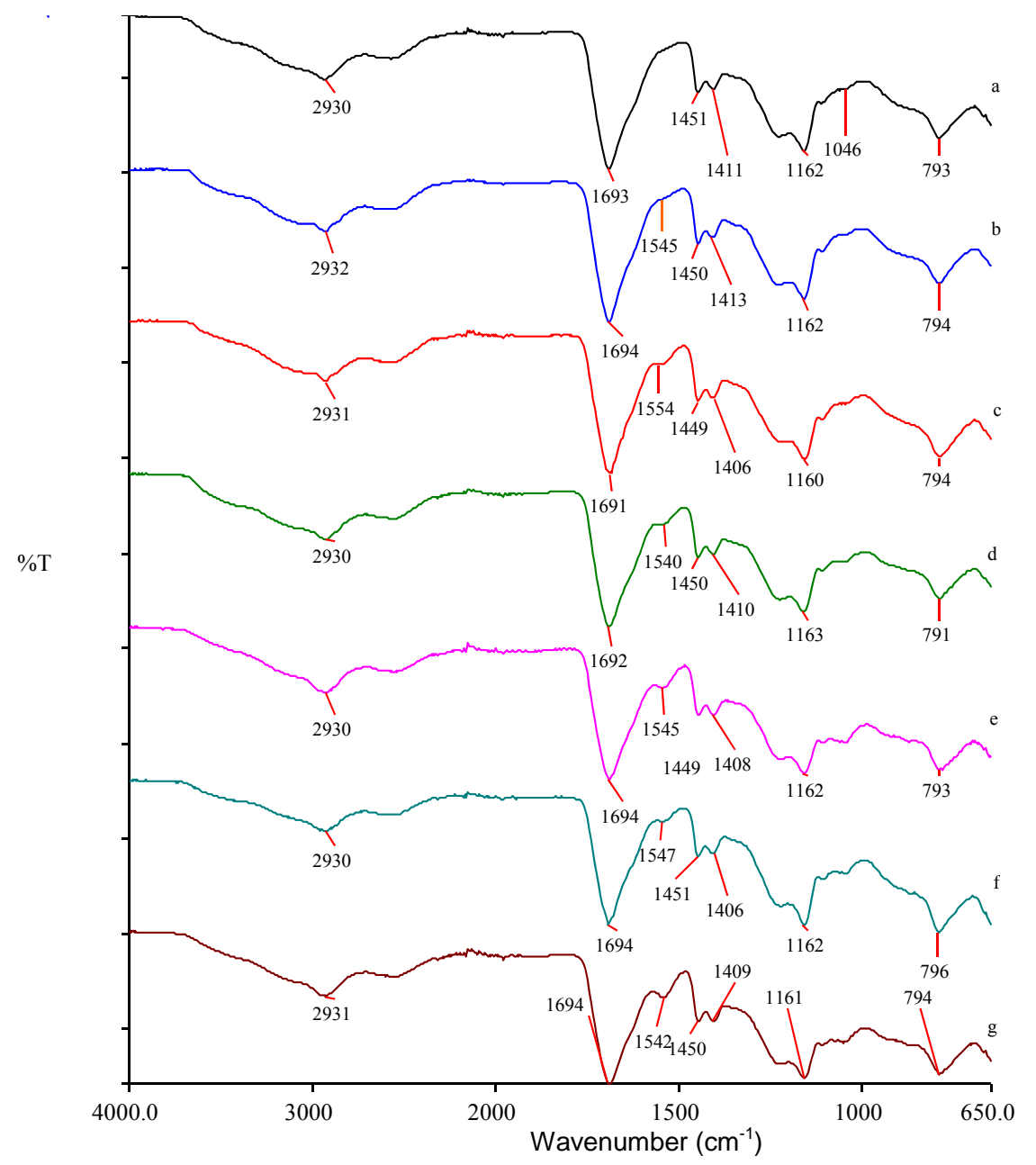

Fig. 2. FTIR-ATR spectrum of a. PAA, b. PAA/3-APT-nHAp(1\%), c. PAA/3-APT-nHAp(2.5\%), d. PAA/3-CPTMSnHAp(1\%), e. PAA/3-CPTMS-nHAp(2.5\%), f. PAA/N-3-TMPEDA-nHAp(1\%) and g. PAA/N-3-TMPEDA$n \mathrm{HAp}(2.5 \%)$ 
When nHAp was modified by 3-APT, N-3-TMPEDA and 3-CPTMS, it was appeared new peaks in FTIR-ATR spectra of OM-nHAp samples. In Figure 1, the difference observed in the vibration frequencies of FTIR-ATR spectrum of 3-APT was considered as the indicator of the interaction between the modifying molecule and nHAp particles. The band at $2928 \mathrm{~cm}^{-}$ , and the band at $1568 \mathrm{~cm}^{-1}$ for 3-APT-nHAp could be assigned to the N-H stretching vibration and to the $\mathrm{NH}_{2}$ deformation mode of free amino groups, respectively. The careful inspection of the $\mathrm{C}-\mathrm{H}$ vibrations for the methoxy and chain $\mathrm{CH}_{2}$ groups at 2928 and $2849 \mathrm{~cm}^{-1}$ for 3-APT was the evident for the modification. The peak due to the chain at $2928 \mathrm{~cm}^{-1}$ was clearly seen while the $\mathrm{C}-\mathrm{H}$ due to the $\mathrm{O}-\mathrm{CH}_{3}$ at $2849 \mathrm{~cm}^{-1}$ disappeared. Because there were no $\mathrm{C}-\mathrm{H}$ bond on the pure nHAp the change in the $\mathrm{C}-\mathrm{H}$ vibrations on the modified structure might be the best evidence for the modification of 3-APT. The change in Si-O stretching at 1469 and $1087 \mathrm{~cm}^{-1}$ might be attributed to the effect of the modification or the modification process [17]. In this case, the reaction between nHAp and 3-APT can be given by the following equation (1).<smiles>CCO[Si](CN)(OCC)OCC</smiles>

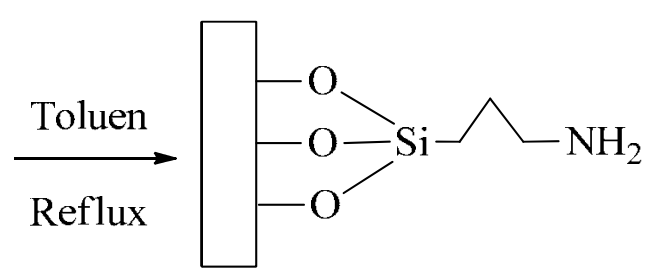

Figure 1 has shown FTIR-ATR spectra for the modification of nHAp with 3-CPTMS. The peak due to methoxy at $2841 \mathrm{~cm}^{-1}$ of 3-CPTMS disappeared as a result of modification. This showed that the modification was a chemical reaction not a physical interaction. Again, the peak in $2944 \mathrm{~cm}^{-1}$ due to C-H stretching vibration of 3-CPTMS shifted at $2957 \mathrm{~cm}^{-1}$ after modification. The modification of nHAp with N-3-TMPEDA understood from FTIR-ATR spectra in Figure 1. The peak at $2949 \mathrm{~cm}^{-1}$ due to alkyl group of modifier shifted at $2924 \mathrm{~cm}^{-1}$ in modified nHAp. Furthermore, the C-N peaks of modifier at 1458 and $1303 \mathrm{~cm}^{-1}$ have shifted at 1472 and $1320 \mathrm{~cm}^{-1}$ after modification.

The FTIR spectrum of PAA at room temperature (Fig. 2a) had a prominent band at 1693 $\mathrm{cm}^{-1}$ associated with $\mathrm{C}=\mathrm{O}$ stretching. In addition, there were distinctive shoulders between 2700 and $2500 \mathrm{~cm}^{-1}$ from overtones and combinations of the C-O stretch band at 1200-1315 $\mathrm{cm}^{-1}$ and from in-plane deformation of C-O-H at 1451 and $1411 \mathrm{~cm}^{-1}$. The wide band at 793 $\mathrm{cm}^{-1}$ was associated with an out-of-plane $\mathrm{OH}$... O deformation indicating the existence of strong interchain hydrogen bonds [24]. FTIR-ATR spectra of PAA/OM-nHAp nanocomposites displayed a new band at about $1547 \mathrm{~cm}^{-1}$. This band was assigned to the formation of chelation complex between carboxylic group of PAA and $\mathrm{Ca}$ ion from OMnHAp which increased with adding OM-nHAp [13]. Also, the carboxyl group band undergoed a slight shift (from $1691 \mathrm{~cm}^{-1}$ to $1694 \mathrm{~cm}^{-1}$ ). This may be due to hydrogen bond between $\mathrm{C}=\mathrm{O}$ group of PAA and $\mathrm{OH}$ group of OM-nHAp. These changes in the carboxyl group indicated that anhydride-like structures were formed. Again, it was observed the band of PAA at $1043 \mathrm{~cm}^{-1}$ in the case of nanocomposite. 


\section{XRD Analysis of OM-nHAps and their nanocomposites}

Figure 3 shows the X-ray diffraction pattern of PAA, nHAp, OM-nHAp and their nanocomposites. The existence of $2 \theta$ peaks at approximately $31.42,32.58$, and $33.82^{\circ}$ corresponding to the diffraction planes (211), (210), and (300) of the HAp crystallites, respectively, confirmed the presence of nHAp [11]. The adding of OM-nHAp powders into PAA as a matrix thus provided an effective means to produce nanocomposites. The OMnHAp samples had characteristic peaks at $2 \theta=26^{\circ}$. As shown in Figure 3, XRD peak at $2 \theta=26^{\circ}$ of OM-nHAp compounds did not appear in the case of nanocomposite. This may be due to low intensity of OM-nHAp into polymer matrix or participating of OM-nHAp in bonding with the polymer or molecular interactions between OM-nHAp and polymer as well [25]. All of PAA/OM-nHAp nanocomposites were homogen distribution as seen from their XRD pattern (Figure 3). At the same time, XRD results were consistent with FTIR results.

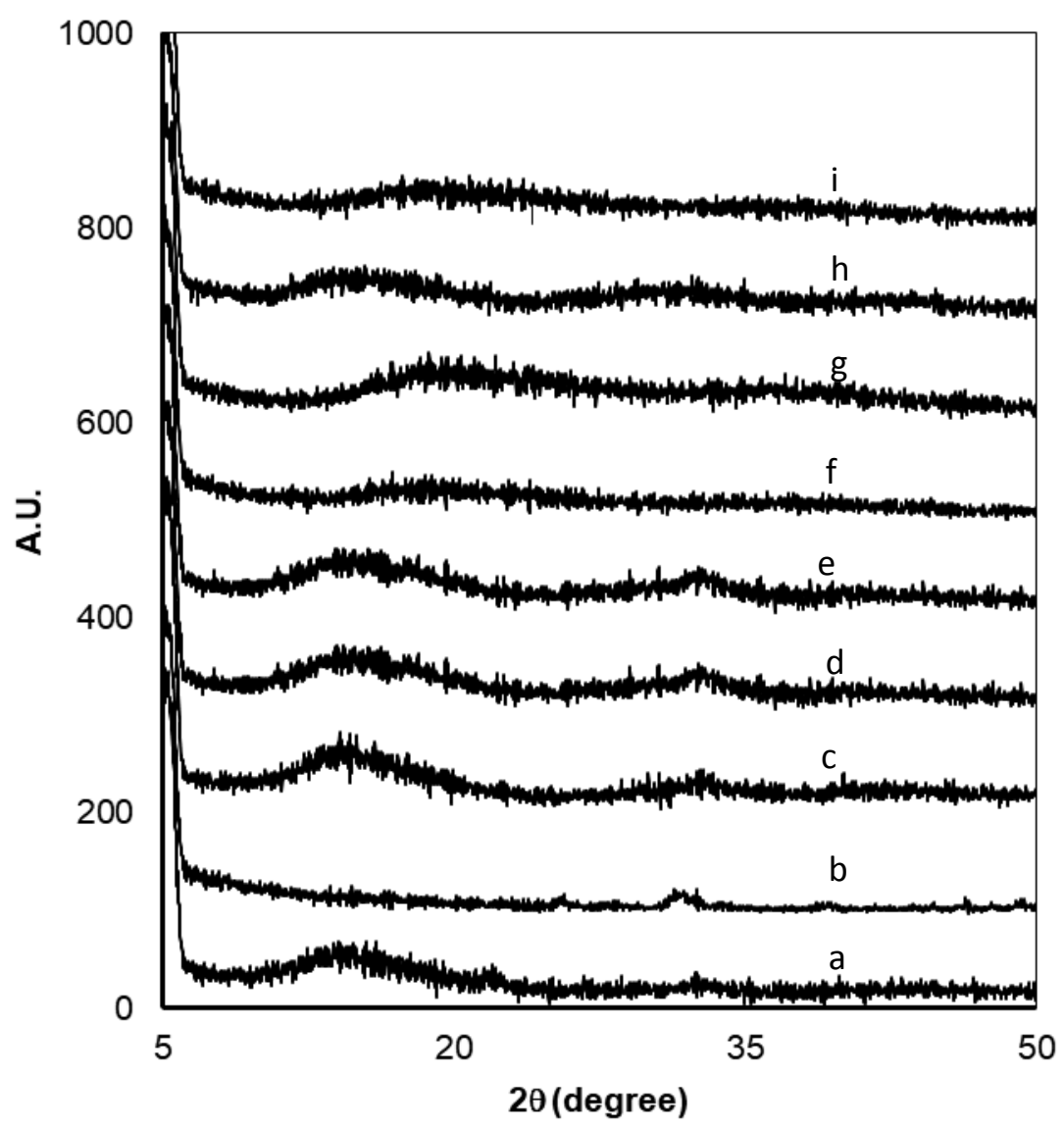

Fig. 3. XRD pattern of a. PAA, b. nHAp, c. PAA/3-APT-nHAp(1\%), d. PAA/3-APT-nHAp(2.5\%), e. PAA/3-CPTMSnHAp(1\%), f. PAA/3-CPTMS-nHAp(2.5\%), g. PAA/N-3-TMPEDA-nHAp(1\%) and h. PAA/N-3-TMPEDA-

\section{SEM and TEM} $\mathrm{nHAp}(2.5 \%)$

The degrees of dispersion of OM-nHAp particles in PAA matrix were represented by TEM and SEM analysis. Figures 4 and 5 have shown SEM and TEM micrographs, respectively. As seen from SEM and TEM micrographs, OM-nHAp particles were in the nanoscale and had a homogeneous distribution in PAA matrix. 

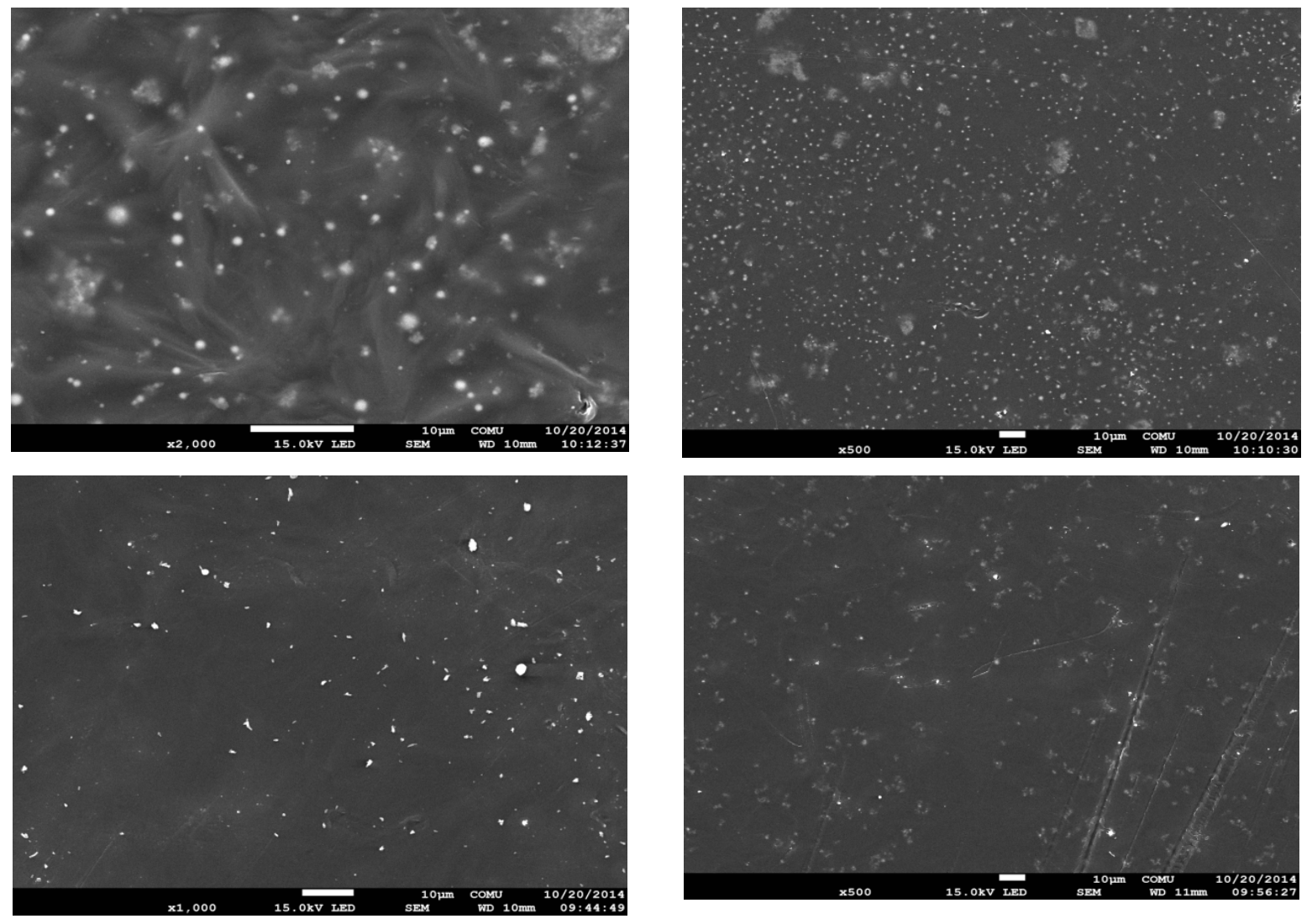

Fig. 4. SEM micrographs of PAA/OM-nHAp nanocomposites

\section{Thermal analysis}

Thermal gravimetric analysis (TG) were used to compare the thermal decomposition of PAA and its nanocomposites, a behavior that directly showed to the dispersion of OM-nHAp into PAA matrix. The importance of this analysis for nanocomposite materials was to measure whether the thermal stability of the polymer improved. Furthermore, it can also be determined the onset temperature of thermal degradation, the temperature of maximum mass loss, and the residue amount. In nanocomposite case, polymer matrices reinforced with small quantities of nano-sized particles can be exhibited difference in its degradation mechanisms. This changes in thermal stability determined with this technical [26]. TG curves of PAA and its nanocomposites were taken under $\mathrm{N}_{2}$ atmosphere at $10{ }^{\circ} \mathrm{C} / \mathrm{min}$ (not shown figures). Thermal stability of OM-nHAp particles is of major importance in controlling the sintering conditions and developing OM-nHAp-reinforced composites. From the results, no significant weight loss was detected for OM-nHAp over the measured temperature range, indicating the high thermal stability of the particles. 

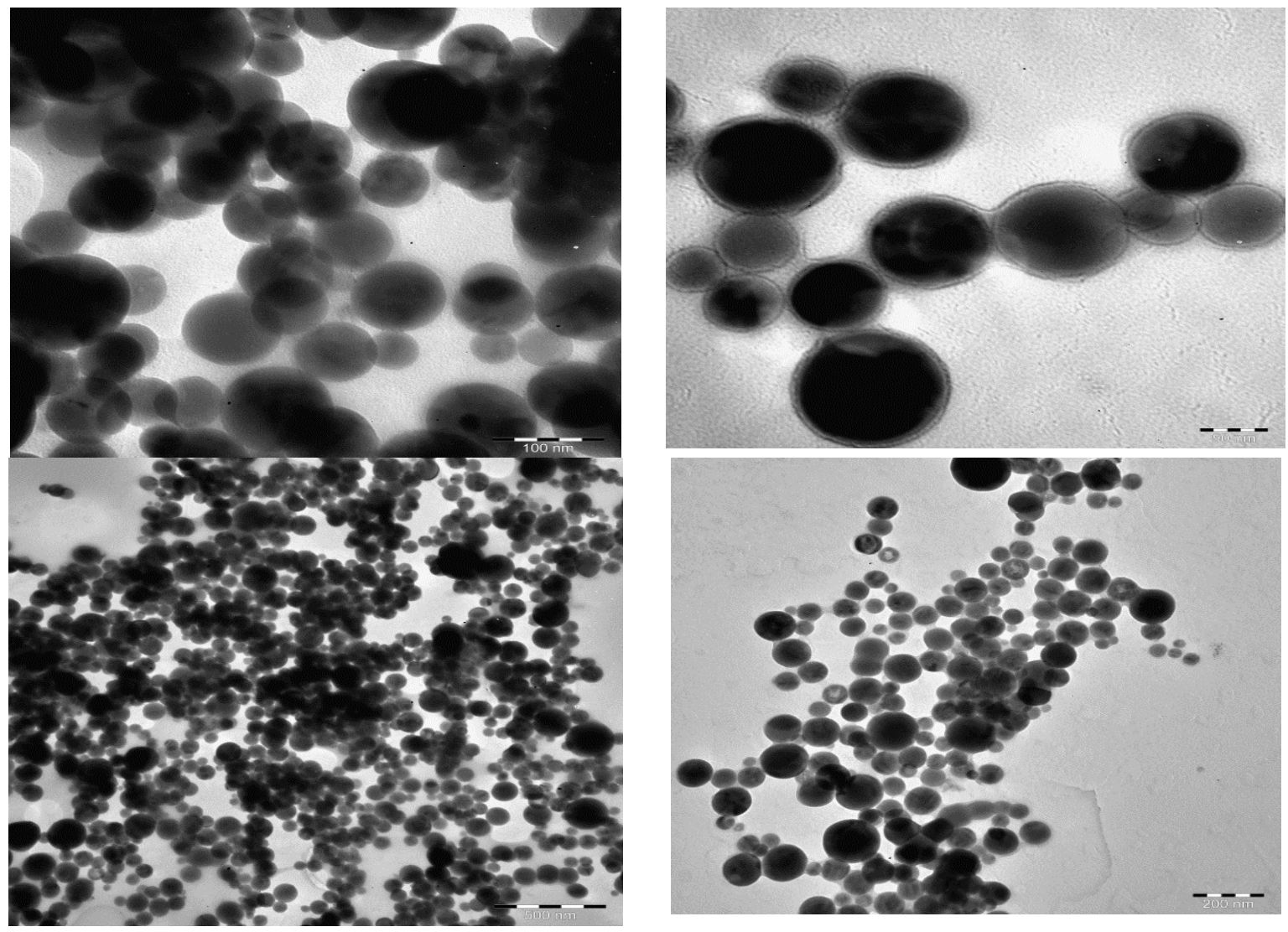

Fig. 5. TEM images of PAA/OM-nHAp nanocomposites

As seen from $\mathrm{TG}$ data in Table 1, the thermal degradation of PAA and its nanocomposites occured through three decomposition steps; dehydration, decarboxylation and chain scission [27]. The temperatures at the maximum weight losses of PAA film were observed at 114,290 and $407{ }^{0} \mathrm{C}$. Step of dehydration could come true by intra or intermolecular reaction of carboxyl groups. The first weight loss in the temperature range of 80-200 ${ }^{\circ} \mathrm{C}$ was due to the evaporation of physically weakly and chemically strongly bound $\mathrm{H}_{2} \mathrm{O}$. As seen from Table 1, the dehydration temperature of PAA for nanocomposite films generally shifted to higher temperatures in the temperature range of $80-200{ }^{\circ} \mathrm{C}$. This showed that the existence of OM-nHAp in the polymer matrix prevented the dehydration of PAA and organophilic treatment improved the thermal stability of PAA/OM-nHAp nanocomposites, due to better interactions between PAA matrix and OM-nHAp. The initial step in the thermal degradation involves the formation of anhydrides. The majority of these anhydrides involve the formation of six-membered glutaric anhydride rings by the reaction of adjacent acid groups [28]. The decarboxylation to give carbon dioxide becomes increasingly important above $250{ }^{\circ} \mathrm{C}$ and both water and carbon dioxide continue to be evolved on heating up to 500 ${ }^{0} \mathrm{C}$. Separation of $\mathrm{CO}_{2}$ as gas shows the decarboxylation of anhydride structures and ketens, ketones and unsaturated compounds forming intermediate compounds. The results showed that the decarboxylation temperature slightly shifted to lower temperature for all nanocomposites. The fact that decarboxylation temperature shifted to lower temperature may be due to decomposition and deactivation of modifiers such as 3-APT, N-3-TMPEDA and 3CPTMS in OM-nHAp. The equation (2) for decarboxylation step can be written as following: 


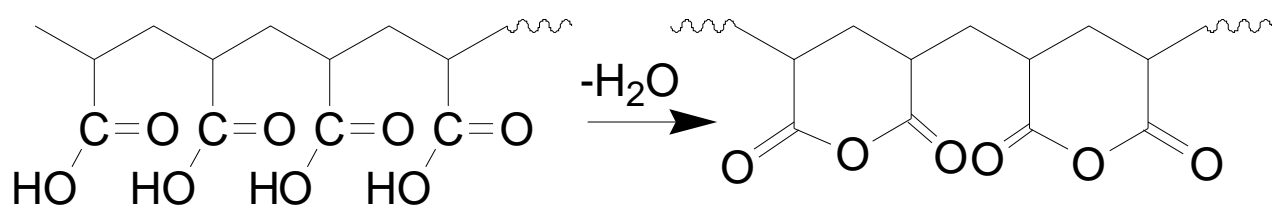

The third step was that chain scission occured at temperature above $350{ }^{\circ} \mathrm{C}$, where the formation of important amounts of cold ring fraction consisting of dimer, trimer, etc. were possible owing to release of fragments with short sequences of acrylic acid [23]. Acrylic acide monomers far away from the structure represented depolymerization reaction [24,27]. The chain scission occurred higher temperature for all nanocomposites. This may be due to the additive effect of OM-nHAp fillers and the formation of chelation complex between carboxylic group of PAA and Ca ion from OM-nHAp, which increased with adding OMnHAp as stated in FTIR-ATR spectra. A similar result was obtained for $\mathrm{PAA} / \mathrm{B}_{2} \mathrm{O}_{3}$ nanocomposites by Beyli et al. (2018) [29]. In their study, both PAA and PAA $/ \mathrm{B}_{2} \mathrm{O}_{3}$ nanocomposites were degraded in three steps. The thermal stability of PAA increased about $10{ }^{\circ} \mathrm{C}$ in the case of nanocomposites. On the contrary, Dubinsky et al. (2004) investigated the thermal decomposition of poly(acrylic acid) (PAAc) containing copper nitrate and they found that the degradation of PAAc/Cu-N was a complex multi-stage process, that adding of $\mathrm{Cu}-\mathrm{N}$ to PAAc caused a dramatic decrease in thermal stability, and that the rate of PAAc/Cu-N degradation was significantly higher in air than in argon [24].

Table 1. TG, DSC and biocompatible results of PAA and its nanocomposites

\begin{tabular}{lccccc}
\hline Samples & $\mathrm{T}_{\max 1}$ & $\mathrm{~T}_{\max 2}$ & $\mathrm{~T}_{\max }$ & $\mathrm{Tg}$ & Hemolysis (\%) \\
\hline PAA & 114 & 290 & 407 & 96 & 36 \\
PAA/3-APT-nHAp (1\% wt.) & 121 & 284 & 434 & 102 & 10 \\
PAA/3-APT-nHAp (2.5\% wt.) & 131 & 271 & 432 & 107 & 4 \\
PAA/N-3-TMPEDA-nHAp (1\% wt.) & 136 & 265 & 436 & 103 & 7 \\
PAA/N-3-TMPEDA-nHAp (2.5\% & 128 & 277 & 438 & 119 & 4 \\
wt.) & 100 & 254 & 434 & 121 & 8 \\
PAA/3-CPTMS-nHAp (1\% wt.) & 162 & 276 & 435 & 117 & 3 \\
PAA/3-CPTMS-nHAp (2.5\% wt.) & & & &
\end{tabular}

DSC is a technique, which allows determining the existence of phase segregation of polymers. Tg values determined by DSC were given in Table 1 for PAA and its nanocomposites. A single glass transition temperature was detected for PAA and its nanocomposites. In the case of nanocomposite, the main transition assigned to the PAA backbone was significantly moved toward higher temperatures. This behavior might be associated with the existence of hydrogen bonds between carboxylic acid groups that lead to an increase of backbone interactions, and between carboxylic acid groups of PAA and functional groups such as $-\mathrm{NH}-,-\mathrm{NH}_{2}$ of modifiers. Such interactions reduced the mobility of the whole polymeric material and, therefore, the occurrence of generalized motions within PAAs shifted to higher temperatures. Another cause may be due to the formation of chelation complex between carboxylic group of PAA and $\mathrm{Ca}$ ion from OM-nHAp, which increased with adding OM-nHAp [13]. Therefore, this chelation formation reduced the mobility of the whole PAA material and Tg increased. 


\section{Optical properties}

The optical properties of PAA/OM-nHAp nanocomposites were evaluated by measuring the optical transmission from ultraviolet to visible using a UV-Vis Lamda 35 spectrophotometer. PAA/OM-nHAp nanocomposite films showed transparency in the range of 200-700 nm. Compared to neat polymer film, the transmittance of the films decreased with the increase of weight content of OM-nHAp nanoparticles, which could be seen in Figure 6. The spectra of films at higher OM-nHAp loading exhibited lower optical clarity, indicating that there was a strong scattering of OM-nHAp resulting in lower transparency of the UV light. As a result, it can be said that the nanocomposites have lower optical transparency than that of PAA film.

\section{Hemocompatibility and biocompatibility}

Table 1 has showed the hemolysis \% of PAA and its nanocomposites using human blood. The degree of hemolysis of blood cells in the presence of PAA was $36 \%$ and it had toxic effect. Similar result was found by Çakir [30]. However, the degree of hemolysis of PAA nanocomposites synthesized using OM-nHAp was lower than that of pure PAA. In particular, the nanocomposites containing OM-nHAp (2.5 wt\%) had high hemocompatibility degree, which the degree of hemolysis was 3.5\%. When OM-nHAp amount increased, hemocompatibility degree of PAA nanocomposites also increased (Table 1). Furthermore, nanocomposites containing OM-nHAp $(2.5 \mathrm{wt} \%)$ was biocompotibility. Similar results were found for PMMA/nHAp nanocomposites by Yilmaz et al. (2018) [9] and for PMMA/3-APTnHAp nanocomposites by Diken et al. (2018) [10]. These results demonstrated that nanocomposites became more biocompatible than polymer by increasing the amount of nHAp and modified-HAp in the PMMA matrix.

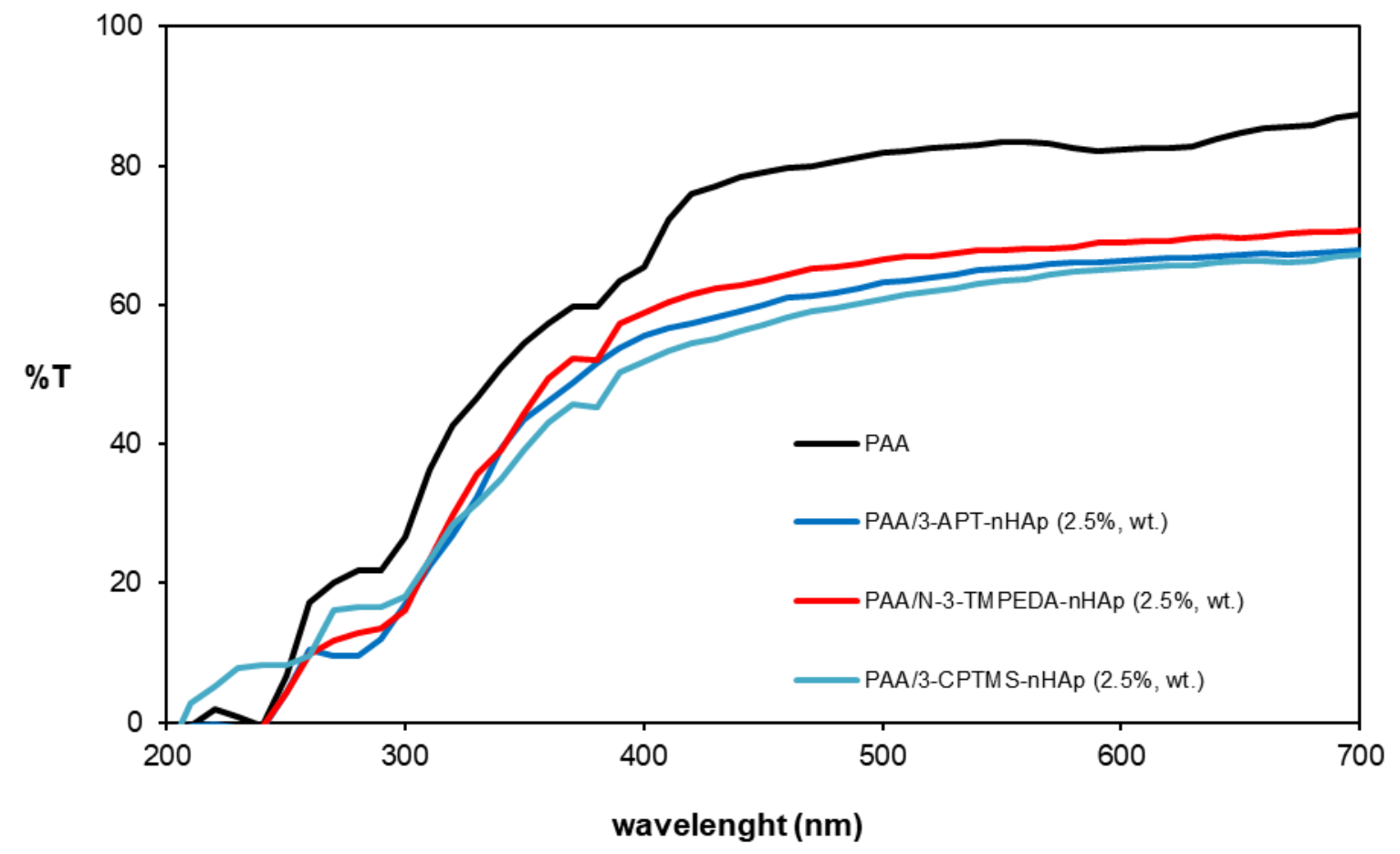

Fig. 6. UV-Vis transmittance spectra of PAA and PAA/OM-nHAp nanocomposites. 


\section{CONCLUSIONS}

PAA/OM-nHAp nanocomposites were prepared by solvent intercalation method. The nanocomposites were characterized by XRD, SEM, TEM, FTIR-ATR, TG, DSC and UVVisible spectrophotometer. The presence of the infrared bands in PAA/OM-nHAp nanocomposite indicated that OM-nHAp was interfered with PAA and the hydrogen bond interactions and the formation of chelation complex between them were occurred. SEM, TEM and XRD revealed that the particle dimension was nanometer regime and that they were uniformly distributed within film. The thermal properties of the synthesized PAA/OM-nHAp nanocomposites were also investigated. The nanocomposites enhanced the formation of residue and improved the thermal stability of the polymer matrix. The nanocomposites exhibited higher glass transition temperature in comparison with pure PAA. The enhanced thermal and glass transition temperature of the composite over the neat PAA indicated the formation of nanocomposites. The results consistently showed that these materials had a good hemocompatibility. Hemolysis of the composites was lower than that of pure PAA.

\section{REFERENCES}

1. Dutta, J., Hofmann, H. in: H.S. Nalwa (Ed.), in: Encyclopedia of Nanoscience and Nanotechnology, vol. 9, American Scientific Publishers, (2004), 617.

2. Sadat-Shojai, M., Khorasani, M.T., Jamshidi, A., Irani, S. Nano-hydroxyapatite reinforced polyhydroxybutyrate composites: A comprehensive study on the structural and in vitro biological properties. Materials Science and Engineering C 33, (2013), 2776-2787.

3. Pon-On, W., Charoenphandhu, N., Tang, I.M., Teerapornpuntakit, J., Thongbunchoo, J., Krishnamra, N. Biocomposite of hydroxyapatite-titania rods (HApTiR): Physical properties and in vitro study. Materials Science and Engineering C 33, (2013), 251-258.

4. Hamad, M., Carretti E., Dei L., Baglioni P., Aqueous polyacrylic acid based gels: physicochemical properties and application the growth of Whitlockite. JCG. 79 (1986), 192-197.

5. Batin, G., Popa, C., Brânduşan, L., Vida-Simiti, I. Mechanical properties of Ti/HA functionally graded materials for hard tissue replacement. Powder Metallurgy Progress, 11(3-4) (2011).

6. Silva, V.V., Lameiras, F.S., Domingues, R.Z. Microstructural and mechanical study of zirconiahydroxyapatite $(\mathrm{ZH})$ composite ceramics for biomedical applications. Composites Science and Technology. 61 (2001), 301-310.

7. Bertoni, E., Bigi, A., Falini, G., Panzavolta, S., Roveri, N. Hydroxyapatite/polyacrylic acid nanocrystals. J. Mater. Chem. 9 (1999), 779-782.

8. Wosek, J. Fabrication of composite polyurethane/hydroxyapatite scaffolds using solvent-casting salt leaching technique. Advances in Materials Science, 15(1) (2015), 14-20.

9. Yilmaz, B., Doğan, S., Kasımoğulları, S.Ç. Hemocompatibility, cytotoxicity, and genotoxicity of poly(methylmethacrylate)/nanohydroxyapatite nanocomposites synthesized by melt blending method. International Journal of Polymeric Materials and Polymeric Biomaterials. 67(6) 2018 351-360.

10. Diken, M.E., Doğan, S., Turhan, Y., Doğan, M. Biological properties of PMMA/nHAp and PMMA/3-APT-nHAp nanocomposites. International Journal of Polymeric Materials and Polymeric Biomaterials. 67(13) (2018), 783-791. 
11. Yoshida, A., Miyazaki, T., Ishida, E., Ashizuka, M. Preparation of bioactive chitosanhydroxyapatite nanocomposites for bone repair through mechanochemical reaction. Materials Transactions. 45(4) (2004), 994-998.

12. Khan, A.S., Wong, F.S.L., McKay, I.J., Whiley, R.A., Rehman, I.U. Structural, mechanical, and biocompatibility analyses of a novel dental restorative nanocomposite. J. Appl. Polym. Sci. 130 (2013), 439-447.

13. El-Bahy, G.S., Abdelrazek, E.M., Allam, M.A., Hezma, A.M. Characterization of in situ prepared nano-hydroxyapatite/polyacrylic acid (HAp/PAAc) biocomposites. Journal of Applied Polymer Science. 122 (2011), 3270-3276.

14. Darder, M., Colilla, M., Ruiz-Hitzky, E. Biopolymer-Clay Nanocomposites Based on Chitosan Intercalated in Montmorillonite. Chem. Mater. 15(20) (2003), 3774-3780.

15. Wang, S.F., Shen, L., Zhang, W-D., Tong, Y-J. Preparation and mechanical properties of chitosan/carbon nanotubes composites. Biomacromolecules, 6(6) (2005), 3067-3072.

16. Turhan, Y., Turan, P., Doğan, M., Alkan, M., Namli, H. and Demirbaş, Ö. Characterization and adsorption properties of chemically modified sepiolite. Industrial \& Engineering Chemistry Research. 47(6) (2008), 1883-1895.

17. Doğan, M., Turhan, Y., Alkan, M., Namli, H., Turan, P. and Demirbaş, Ö. Functionalized sepiolite for heavy metal ions adsorption. Desalination. 230(1-3) (2008), 248-268.

18. Kızılduman, B.K., Alkan, M., Doğan, M., Turhan, Y. Al-pillared-montmorillonite (AlPMt)/poly(methyl methacrylate)(PMMA) nanocomposites: the effects of solvent types and synthesis methods. Advances in Materials Science, 17(3) (2017), 5-23.

19. Turhan, Y., Doğan, M. and Alkan, M. Poly(vinyl chloride)/kaolinite nanocomposites: Characterization, Thermal and Optical Properties. Industrial \& Engineering Chemistry Research. 49 (2010), 1503-1513.

20. Turhan, Y., Alp, Z.G., Alkan, M., Doğan, M., Preparation and characterization of poly(vinylalcohol)/modified bentonite nanocomposites. Microporous and Mesoporous Materials. 174 (2013), 144-153.

21. Belanger, M, Marois, Y. Hemocompatibility, biocompatibility, inflammatory and in vivo studies of primary reference materials low-density polyethylene and polydimethylsiloxane: a review. Journal of Biomedical Materials Research. 58(5) (2001), 467-477.

22. Arsad, S.M., Lee, P.M., Hunk, L.K. Synthesis and characterization of hydroxyapatite nanoparticles and $\beta$-TCP particles. International Conference on Biotechnology and Food Science. 7 (2011), 184-188.

23. Hojjati, B., Sui, R., Charpentier, P.A. Synthesis of $\mathrm{TiO}_{2} / \mathrm{PAA}$ nanocomposite by RAFT polymerization. Polymer. 48 (2007), 5850-5858.

24. Dubinsky, S., Grader, G.S., Shter, G.E., Silverstein, M.S. Thermal degradation of poly(acrylic acid) containing copper nitrate. Polymer Degradation and Stability. 86 (2004), 171-178.

25. Turhan, Y., Doğan, M., Alkan, M. Characterization and some properties of poly(vinyl chloride)/sepiolite nanocomposites. Advances in Polymer Technology. 32(1) (2013) E65-E82.

26. Corcione, C.E., Frigione, M. Characterization of nanocomposites by thermal analysis. Materials. 5 (2012), 2960-2980.

27. Fuente, J.L., Wilhelm, M., Spiess, H.W., Madruga, E.L., Fernandez-Garcia, M. Cerrada, M.L. Thermal, morphological and rheological characterization of poly(acrylic acid-g-styrene) amphiphilic graft copolymers. Polymer. 46 (2005), 4544-4553. 
28. McGaugh, M.C., Kottle, S. The thermal degradation of poly(acrylic acid). Journal of Polymer Science Part B: Polymer Letters. Journal of Polymer Science Part B: Polymer Letters. 5(9) (1967), 817-820.

29. Beyli, P.T., Doğan, M., Gündüz, Z., Alkan, M., Turhan, Y. Synthesis, characterization and their antimicrobial activities of boron oxide/poly(acrylic acid) nanocomposites: thermal and antimicrobial properties. Advances in Materials Science, 18(1) (2018), 28-36.

30. Çakir, R. The development of a new culture model to study the toxicity of polymers. MSc Thesis. Yildiz Technical University, Istanbul-Turkey, 2008. 